\title{
Slug Flow Model for Infiltration into Fractured Porous Media
}

\author{
M. J. Martinez \\ Engineering Sciences Center \\ Sandia National Laboratories \\ Albuquerque, NM 87185 \\ 505-844-8729, 505-844-4523, mjmarti@sandia.gov
}

\begin{abstract}
A model for transient infiltration into a periodically fractured porous layer is presented. The fracture is treated as a permeablewalled slot and the moisture distribution is in the form of a slug behind an advancing meniscus. The wicking of moisture from the fracture to the unsaturated porous matrix is a nonlinear diffusion process and is approximated by self-similar solutions. The resulting model is a nonlinear Volterra integral equation with a weakly singular kernel. Numerical analysis provides solutions over a wide range of the parameter space and reveals the asymptotic forms of the penetration of this slug in terms of dimensionless variables arising in the model. The numerical solutions corroborate asymptotic results given earlier by Nitao and Buscheck (1991), and by Martinez (1988). Some implications for the transport of liquid in fractured rock are discussed.
\end{abstract}

\section{INTRODUCTION}

Understanding the role of fractures, macropores or worm holes in transporting moisture and contaminants in unsaturated porous materials is of interest in a variety of subsurface flow phenomena. Examining the role played by fractures is essential to the safe design of nuclear waste repositories. The problem is also similar to models for flow through macropores (Bevin and Germann, 1982), where the role of macropores on flow of moisture and fertilizers is of interest. The problem considered here is similar to the theory discussed by Philip (1968) regarding flow in aggregated media, the major difference is the neglect of gravity in Philip's model.

Apparently the first application of this slug model to flow in fractured rock was by Martinez (1988), followed shortly thereafter by Nitao and Buscheck (1991). Martinez presented numerically generated solutions for a large range of fracture to matrix conductivity ratios. However, the solutions were restricted to relatively short timescales, so the long-time limits were not discussed. The timescales were thought to be representative of timescales for summer thunderstorms in arid regions. Following Philip (1968), Nitao and Buscheck (1991) performed an asymptotic analysis of the slug model, and determined a series of asymptotic penetration rates for various timespans over the history of infiltration.

In this paper, we extend the slug model described by Martinez (1988) to include the long-time limit, and therefore, interference effects on matrix absorption from neighboring fractures. This includes development of a matrix absorption model for finitesize blocks. The efficacy of the proposed absorption model is demonstrated by comparison with the numerical solution of the full nonlinear absorption problem. The numerical solutions of the present model recover the asymptotic penetration rates predicted by Nitao and Buscheck (1991); the numerical results presented here show the full variation of the solutions with respect to system parameters, and provide solutions for the cumulative flux imbibed by the system in addition to the slug penetrations.

\section{SLUG MODEL EQUATIONS}

In the slug model, the fracture is considered to have a very narrow range of characteristic pore-size as compared to the surrounding porous material. As a consequence the moisture penetration in the fracture is approximated as a slug with a sharp leading edge. That is, if the location of the front is at $z=\zeta(t)$, where $z$ is the distance along the fracture, then, $s_{f}=1$ for $z<\zeta$, 


\section{DISCLAIMER}

This report was prepared as an account of work sponsored by an agency of the United States Government. Neither the United States Government nor any agency thereof, nor any of their employees, make any warranty, express or implied, or assumes any legal liability or responsibility for the accuracy, completeness, or usefuiness of any information, apparatus, product, or process disclosed, or represents that its use would not infringe privately owned rights. Reference herein to any specific commercial product, process, or service by trade name, trademark, manufacturer, or otherwise does not necessarily constitute or imply its endorsement, recommendation, or favoring by the United States Government or any agency thereof. The views and opinions of authors expressed herein do not necessarily state or reflect those of the United States Government or any agency thereof. 


\section{DISCLAIMER}

Portions of this document may be illegible in electronic image products. Images are produced from the best available original document. 


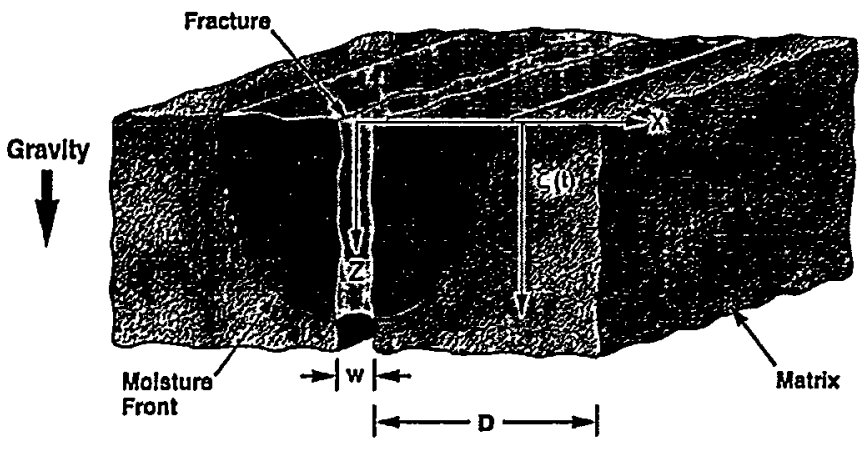

Figure 1. Schematic depicting the penetration of a liquid slug into a fracture embedded in an unsaturated matrix.

and $S_{f}=S_{f}\left(\Psi_{\infty}\right)$ for $z>\zeta$, where $S_{f}$ is the moisture saturation in the fracture, and $\psi_{\infty}$ is the initial pressure head (sometimes

called matric potential) in the material, see Fig. 1. Furthermore, the moisture absorption into the surrounding porous material is assumed to be locally perpendicular to the fracture, that is, flow along the direction of the fracture in the porous block is not considered.

Under these assumptions, the flow in the fracture is described by a moving boundary problem,

$$
-K_{f} \frac{\partial^{2} h}{\partial z^{2}}+\frac{2 q_{w}}{w}=0, \quad z<\zeta(t),
$$

where $K_{f}=\left(\rho g k_{f}\right) / \mu$ is the fracture conductivity, $\rho$ is density, $g$ is gravity, $k_{f}$ is intrinsic permeability, $\mu$ is the dynamic viscosity, $h(z, t)=\psi-z$ is the piezometric head, $w$ is the effective fracture aperture, and $q_{w}(z, t)$ is the absorption flux

from the fracture to the matrix at elevation $z$ in the fracture. As indicated, this flow equation is valid in the region behind the advancing moisture front and is subject to the following boundary conditions,

$$
\begin{gathered}
h(0, t)=h_{0}(t) \\
h(\zeta, t)=\Psi_{c}-\zeta(t)=h_{\zeta}
\end{gathered}
$$

where $\psi_{c}$ is the value of pressure head just behind the front.

Because Eqn. (1) does not involve time explicitly, it can be solved $a$ priori for a general source function $q_{w}(z, t)$. The solution can be obtained through the use of a Green's function, which can be used to solve Eqn. (1) for a distributed source (Stakgold, 1979). Then, the solution to (1) subject to (2) is given by superposing the homogeneous solution and the product of the Green's function and the source, yielding,

$$
h(z, t)-h_{0}=\left(h_{\zeta}-h_{0}\right) \frac{z}{\zeta}+\int_{0}^{\zeta(t)} G(z, \xi) \frac{2 q_{w}}{w K_{f}} d \xi
$$

where $G$ is the well-known Green's function of Eqn. (1),

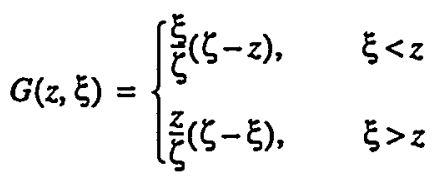

where $\xi$ is the source point of the Green's function. The location of the front $\zeta(t)$ is determined by balancing the flux of material at the interface,

$$
\phi_{f}\left(1-S_{f \infty o}\right) \frac{d \zeta}{d t}=-\left.K_{f} \frac{\partial h}{\partial z}\right|_{z=\zeta}
$$

where $\phi_{f}$ is fracture porosity, $S_{f o 0}$ is the initial moisture saturation in the fracture. Note that if the fracture is approximated by a slot with aperture $w$, then $\phi_{f}=1$ and $S_{f \infty}=0$, and the characteristic capillary pressure is $\Psi_{c}=2 \sigma \rho g / w$, where $\sigma$ is the surface tension. Using Eqn. (3) in the flux balance results in a nonlinear integro-differential equation for the penetration history,

$$
\phi_{f}\left(1-S_{f \infty}\right) \frac{d \zeta}{d t}=K_{f}\left(1+\frac{\Psi_{c}}{\zeta}\right)-\frac{2}{w \zeta} \int_{0}^{\zeta} q_{w}\left(t-s\left(\zeta^{\prime}\right)\right) \zeta^{\prime} d \zeta^{\prime},
$$

where $s(\zeta)=t=\zeta^{-1}(t)$ i.e., the inverse of the desired solution $\zeta(t)$. Once $\zeta(t)$ has been determined, the solution for $h(t)$ can be computed by quadrature according to Eqn. (3), and the flux can also be determined by differentiation.

When the surrounding matrix material is impermeable, i.e. $q_{w}=0$, the penetration equation is separable and has the solution,

$$
\tau=L-\log (1+L), \quad L=\frac{\zeta}{\Psi_{c}}, \quad \tau=\frac{K_{f}}{\Psi_{c} \phi_{f}\left(1-S_{f \infty o}\right)} .
$$

Using the dimensionless variables indicated above, the nondimensional equation for the penetration is

$$
\frac{d L}{d t}=1+\frac{1}{L}-\frac{1}{L} \int_{0}^{L} q\left(\tau-s\left(L^{\prime}\right)\right) L^{\prime} d L^{\prime}, \quad q=\frac{2 q_{w}}{K_{f}} \frac{\Psi_{c}}{w}
$$

where $q$ is the dimensionless absorption flux from the fracture to matrix, which depends only on the elapsed time since the moisture front passed the location $z$ along the fracture. The penetration equation is subject to $L(0)=0$. This equation is a nonlinear Volterra integral equation of the second kind (Baker, 1977) and in general requires numerical treatment.

Implicit in the slot model is that the absorption can be described by a function of the form $q_{w}(z, t)$, describing the flux of fluid from the fracture to matrix at elevation $z$ and time $t$. The 
absorption problem is self-similar initially. However, for times which are long enough, interaction of absorption from neighboring fractures must be considered. The absorption model considered here is discussed in the following section.

\section{ABSORPTION MODEL}

In the slot model, the motion of the slug of moisture in the fracture creates a step-jump boundary condition for the onedimensional lateral absorption of moisture by the porous material at elevation $z$. Hence, the absorption problem can be stated as,

$$
\frac{d \theta_{m}}{d \psi} \frac{\partial \psi}{\partial t}=\frac{\partial}{\partial x}\left(K_{m} \frac{\partial \psi}{\partial x}\right)
$$

where $\theta_{m}$ is the volumetric moisture content in the matrix. The initial and boundary conditions are,

$$
\psi(x, z, 0)=\psi_{\infty} \quad \psi\left(\frac{w}{2}, z, t\right)=\psi_{f}(z, t) \quad \frac{\partial \psi}{\partial x}(D, t)=0
$$

where $\Psi_{f}$ denotes the pressure head in the fracture, and $D$ is the fracture half-spacing. The pressure head is of course continuous and hence no distinction between its value in the fracture or matrix is necessary. However, the distinction is made here for clarity in the discussion. Owing to the slug approximation in the slot model, the middle condition is replaced with $\psi(w / 2, z, t)=\psi\left(\phi_{m} S_{w}\right)$ where $\phi_{m}$ denotes matrix porosity and $S_{w}$ is the matrix saturation at the fracture interface (usually assumed to be unity). In the following, the matrix is assumed saturated at the fracture/matrix interface when the fracture slug penetrates the elevation $z$. Under these conditions, the absorption problem can be solved $a$ priori once the matrix material functions are specified. This is because the matrix absorption at elevation $z$ depends only on the elapsed time since the passage of the moisture slug, hence this one-dimensional absorption problem is well-posed and can be solved independently of the fracture moisture problem. This leads to convolution integrals in the fracture penetration equation in order to account for the variable absorption by the matrix over the length of fracture.

\section{Semi-Infinite Fracture Spacing}

If the fracture spacing is large enough that over the time of interest the moisture at $x=D$ remains at its initial value, the absorption problem is self-similar. Note that the problem is always self-similar for early times in the absorption history. This problem was discussed by Martinez (1988), where solutions are given for various materials representative of the tuff units in Yucca Mountain. These solutions were obtained by recognizing the absorption to be self-similar; therefore the partial differential equation for $\Psi(x, t)$ can be transformed into an ordinary differential equation and solved by a shooting method.

We will not reiterate the entire development here, and instead refer interested readers to Martinez (1988). However, we summarize the major results. By introducing the similarity variable

$$
\eta=\frac{x}{2\left(\frac{K_{m} t}{\alpha_{m}\left(\beta_{m}-1\right) \phi_{m}\left(S_{w}-S_{m r}\right)}\right)^{1 / 2}}
$$

the absorption equation (8) is reduced to a second-order nonlinear ordinary differential equation (ODE) for $\psi(\eta)$. The constants $\alpha_{m}$ and $\beta_{m}$ are material parameters in the vanGenuchten (van Genuchten, 1980) model for matrix moisture retention and relative permeability functions. The ODE can be solved for various values of initial matrix saturation, boundary saturation, and material parameters, see Martinez (1988).

The most important result is that the absorption flux at the fracture/matrix interface is given by

$$
q_{w}=\frac{y_{0}}{\sqrt{t}}
$$

where $v_{0}$ embodies the various material parameters and initial and boundary saturations, and is twice the so-called sorptivity for the matrix material as defined by Philip (1969). Of special note is the simple variation with time of the absorption flux. For the van-Genuchten model used here, $v_{0}$ takes the form,

$$
v_{0}=\frac{1}{2} \alpha_{m}\left(\frac{K_{m}\left(\beta_{m}-1\right) \phi_{m}\left(S_{w}-S_{m r}\right)}{\alpha_{m}}\right)^{\frac{1}{2}}\left(-\left.\frac{d \psi}{d \eta}\right|_{\eta=0}\right)
$$

Here $S_{w}$ denotes the matrix saturation at the fracture/matrix interface, which we take as unity in the present problem, $S_{m r}$ denotes the residual saturation for the matrix material, and the boundary gradient $d \psi / d \eta$ is determined from the numerical solution of the resulting nonlinear ODE. All material models involve a characteristic capillary pressure; it is $1 / \alpha_{m}$ (in units of pressure head) in the van Genuchten model, the bubbling pressure in the Brooks and Corey (1966) model, and $1 / \alpha_{G}$ in the exponential model of Gardner (1958), $k_{r m}=\exp \left(\alpha_{G} \Psi\right)$. Hence, regardless of which material model is used for the matrix, a characteristic diffusivity for the self-similar absorption problem will appear in the absorption flux expression.

\section{Finite Fracture Spacing}

If the absorption process is long enough, there will be interaction between moisture absorption from neighboring 
fractures and the above analysis requires modification. In this case the full problem described in (8) and (9) must be considered. Early in time the process is self-similar with solution as in Eqn. (11). The absorption rate in a linear problem (heat conduction) decreases exponentially in time when the symmetry boundary is important, see for example Carslaw and Jaeger, (1959). In the present nonlinear absorption process, solutions are self-sharpening because the hydraulic conductivity increases with moisture content. Hence, the absorption rate is expected to decrease faster than exponentially once the symmetry boundary is approached. In view of these considerations, rather than attempting a solution of (8), we assume the approximate form,

$$
q_{w}=\left\{\begin{array}{rc}
\frac{v_{0}}{\sqrt{t}}, & t<t_{D} \\
\frac{v_{0}}{\sqrt{t_{D}}} \exp \left(-\frac{t^{n}-t_{D}^{n}}{2 n t_{D}^{n}}\right), & t>t_{D}
\end{array}\right.
$$

This form is exact for $t<t_{D}$ and ensures that the flux and its derivative with time are continuous at $t=t_{D}$. The value of $t_{D}$ is determined by requiring that

$$
\int_{0}^{\infty} q_{w}(z, t) d t=D \phi_{m}\left(S_{w}-S_{m \infty}\right)
$$

which results in,

$$
t_{D}=\left[\frac{D \phi_{m}\left(S_{w}-S_{m \infty}\right)}{v_{0}\left(2+(2 n)^{(1-n) / n} e^{1 / 2 n} I(n)\right)}\right]^{2}
$$

where $S_{m \infty}$ is the initial saturation in the matrix material, $n$ is a free parameter and

$$
I(n)=\int_{(2 n)^{-1 / n}}^{\infty} e^{-\xi^{n}} d \xi .
$$

The value of $n$ determines the rate at which the absorption decreases with time due to the finite fracture spacing. For very large $n$, the absorption model behaves much like a cut-off time, wherein the absorption rate jumps from a finite value to zero at $t=t_{D}$.

The absorption model was verified by comparison to finite difference numerical solutions of the absorption problem for a finite depth layer of volcanic tuff material. The comparison was made for $1 \leq n \leq 6$. For example, the exponential decay $(n=1)$ under-estimates the decay rate past the self-similar regime and under-estimates the fill-time. Larger values of $n$ improve the comparison with the numerical solution. We find the value $n=6$ yields a good comparison of the decay rate of the absorption once the symmetry boundary is important. Furthermore, parameter studies with various $n$ in the slug flow solutions to be discussed presently indicate that values of $n \geq 2$ are sufficient.

\section{INFILTRATION INTO FRACTURED MEDIA}

The absorption model presented in the foregoing completes the specification of the slug flow model. In terms of dimensionless variables given earlier, the dimensionless flux can be written in the form,

$$
q=\left\{\begin{aligned}
\frac{\lambda}{\sqrt{\tau-s(L)}}, & \tau-s(L)<\tau_{D} \\
\frac{\lambda}{\sqrt{\tau_{D}}} \exp \left(\frac{\tau_{D}^{n}-(\tau-s(L))^{n}}{2 n \tau_{D}^{n}}\right), & \tau-s(L)>\tau_{D}
\end{aligned}\right.
$$

where $s(L)$ is the inverse of the penetration function. This equation explicitly shows that the slug model is a threeparameter problem, involving $\tau_{D}, n$, and a dimensionless matrix absorption parameter,

$$
\lambda=\frac{\Psi_{c}}{w}\left(\frac{D_{m} \phi_{m}\left(1-S_{m \infty}\right)}{D_{f} \phi_{f}\left(1-S_{f \infty}\right)}\right)^{1 / 2}\left(-\left.\frac{d \Psi}{d \eta}\right|_{\eta=0}\right),
$$

where $D_{f}=K_{f} \psi_{c}$ and $D_{m}=K_{m} / \alpha_{m}$ are fracture and matrix diffusivities. The parameter $\lambda$ has the significance of being a dimensionless absorption flux, small values indicating very low matrix diffusivity compared to fracture diffusivity.

In general, the penetration equation is a nonlinear integrodifferential equation and solutions were obtained by numerical methods. The numerical treatment is discussed in the appendix. Verification of the algorithm was accomplished by comparing with the known solutions for an impermeable matrix, $\lambda=0$, and the analytical solution for the gravity-free problems.

\section{Semi-Infinite Fracture Spacing}

Fig. 2 shows numerical solutions for $L(\tau)$ for various values of $\lambda$. Plotted on log-log coordinates, two asymptotic time regimes are evident. For very early time, $\tau \ll \lambda^{-2}$, flow into the fracture is dominated by very large pressure gradients owing to capillary forces, with matrix imbibition a sub-dominant effect. The capillary force term in the penetration equation (7) is represented by the $1 / L$ factor, which initially dominates the penetration rate since $L(0)=0$. In this "loss-free" regime,

$$
L-\sqrt{2 \tau}, \quad \tau \ll \lambda^{-2},
$$

independent of $\lambda$; this form can be obtained from the solution in Eqn. (6). For $\tau \gg \lambda^{-2}, L \sim \sqrt{\tau}$ as well, although the solution is not independent of $\lambda$. Hence, the penetration rate is of the same form for very early time and for late time, with an intermediate 


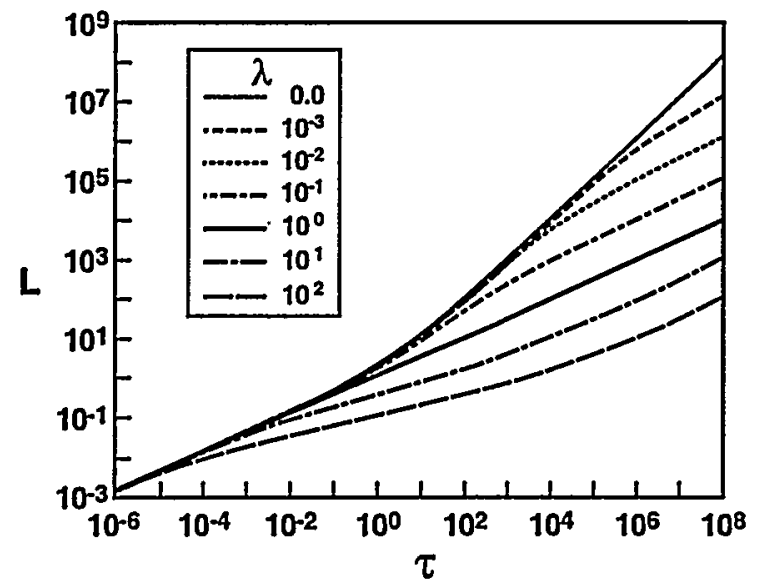

Figure 2. Slug penetration history for semi-infinite fracture spacing.

zone in which the rate depends on $\lambda$. In particular, the solutions show that the late-time asymptotic form is

$$
\frac{\lambda L}{\sqrt{\tau}}=\text { constant }
$$

for finite $\lambda$.

There is a particular solution, $\lambda L^{*}=\sqrt{\tau}$, for the special value $\lambda=\lambda^{*}=1 / \sqrt{2}$. This is an exact solution of Eqn. (7) for all $\tau$ for the case of semi-infinite fracture spacing. This solution would plot as a straight line on Fig. 2. Hence, this solution determines the constant in Eqn. (19) above as unity, and is valid for $\tau \gg \lambda^{-2}$ over the range of $\lambda$ shown in Fig. 2. The two asymptotic limits mentioned above for very small and very large $\tau$ also plot as straight lines, both with slopes of $1 / 2$. The transition zone between these two limits is concave upward for $\lambda<\lambda^{*}$, where the penetration rate increases faster than $\sqrt{\tau}$, and concave downward for $\lambda>\lambda^{*}$ before returning to the $\sqrt{\tau}$ rate at late-time. When $\lambda \ll \lambda^{*}$ the rate of penetration of the slug is much faster than the rate of absorption by the matrix, and the penetration rate increases from $\sqrt{\tau}$ to a rate bounded by $\tau$, achieved in the completely loss-free limit $(\lambda=0)$. When $\lambda » \lambda^{*}$, the rate of fall is much slower than the rate of absorption by the matrix, and hence the penetration rate decreases below $\sqrt{\tau}$ in the transition zone. Nitao and Buscheck (1991) arrived at a bound of $\tau^{1 / 4}$ in this limit. Our numerical results for $\lambda=100$ indicate $L \sim \tau^{0.26}$ in the transition zone $\lambda^{-2} \ll \tau \ll \lambda^{2}$.

Asymptotic analysis of the penetration equation was performed by Nitao and Buscheck (1991). The numerical results discussed above for the loss-free and late-time asymptotes confirm the results of Nitao and Buscheck for these bounding values; our numerical solutions give the full variation with respect to the dimensionless parameters.

Also of interest is the cumulative infiltration of moisture into the fracture at the fracture inlet. The nondimensional cumulative infiltration at time $\tau$, is given by

$$
\frac{Q_{0}}{w \Psi_{c}}=L+2 \lambda \int_{0}^{L}(\tau-s(\xi))^{1 / 2} d \xi .
$$

Fig. 3 shows the history of the cumulative flux into the fracture for the same range of $\lambda$ in Fig. 2. The figure shows that $Q_{0}$ is virtually independent of $\lambda$ for $\lambda<\lambda^{*}$. For large loss parameters the cumulative influx increases in the transition region. This increase is a manifestation of the large matrix imbibition compared to capillary forces acting on the slug as discussed in the previous paragraph.

- In general, the cumulative infiltration is computed numerically (see Appendix). However, the asymptotic forms discussed previously can be used to obtain asymptotic limits for $Q_{0}$ as well. In the loss-free regime, the cumulative infiltration is given by

$$
\frac{Q_{0}}{w \Psi_{c}}=\sqrt{2 \tau}\left(1+\frac{\pi}{2} \lambda \sqrt{\tau}\right), \quad \lambda \sqrt{\tau} \ll 1,
$$

obtained by using Eqn. (18) in Eqn. (20). The cumulative infiltration is dominated by the amount of moisture in the fracture, represented by the first term on the RHS of (21). The second term, which represents the amount absorbed by the matrix, is smaller by a factor of $\lambda \sqrt{\tau}$. In the late-time asymptotics, use of (19) in (20), with the constant set equal to unity, gives

$$
\frac{Q_{0}}{w \Psi_{c}}=\lambda^{-1} \sqrt{\tau}+\frac{\pi}{2} \tau, \quad \lambda \sqrt{\tau} \gg 1,
$$

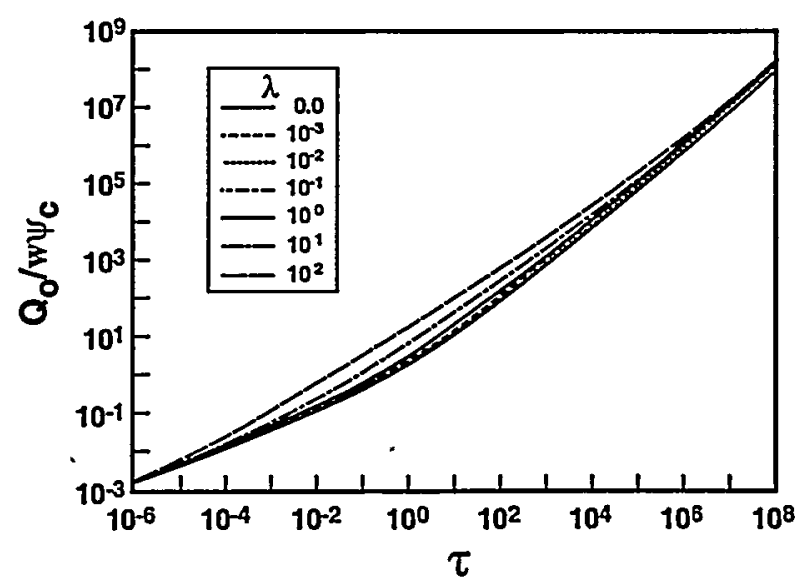

Figure 3. Cumulative absorption of water by the fracture for semi-infinite fracture spacing. 
valid for finite $\lambda$. For $\lambda=0$,

$$
\frac{Q_{0}}{w \Psi_{c}} \approx \tau
$$

in the late-time limit, which is obtained from the analytic result given in Eqn. (6). Note that Eqn. (22) is an exact solution for $\lambda=\lambda^{*}=1 / \sqrt{2}$, and that Eqns. (21) and (22) are identical for this value of $\lambda$. Furthermore, since Eqn. (22) can be written as

$$
\frac{Q_{0}}{w \Psi_{c}}=\frac{\pi}{2} \tau\left(1+\frac{2}{\pi \lambda \sqrt{\tau}}\right)
$$

we note that the cumulative infiltration becomes independent of $\lambda$ for $\tau \gg 4 /(\pi \lambda)^{2}$ and is greater than the loss-free case $(\lambda=0)$ by a factor of $\pi / 2 \approx 1.57$, all in the late-time limit.

\section{Finite Fracture Spacing}

The introduction of finite fracture spacing and the associated absorption model brings $\tau_{D}$ and $n$ as additional parameters into the problem. Comparison of penetration solutions for various values of $n$ and for materials representative of Yucca Mountain indicates that the effect of $n$ on the penetration is weak. This testing included variations in values of $D$ and $S_{m_{\infty}}$. From Eqn. (15), the time for the matrix absorption to be influenced by absorption from neighboring fractures is given by

$$
\tau_{D}=\frac{K_{f} t_{D}}{\Psi_{c}\left(\phi_{f}\left(1-S_{f \infty}\right)\right)}=\left[\frac{2 D \phi_{m}\left(S_{w}-S_{m \infty}\right)}{\lambda w \gamma(n)}\right]^{2}
$$

where

$$
\gamma(n)=2\left[1+(2 n)^{(1-n) / n} e^{1 / 2 n} \Gamma\left(\frac{1}{n}, \frac{1}{2 n}\right)\right]
$$

in terms of the Incomplete Gamma function, $\Gamma(a, x)$

(Abramowitz and Stegun, 1972).

Fig. 4 shows penetration histories for $\tau_{D}=10^{3}$. For $\tau<\tau_{D}$, the solutions are identical to those for semi-infinite fracture spacing, by definition. However, for $\tau>\tau_{D}$, the solutions depart from the semi-infinite curve and eventually attain a constant penetration rate. The numerical solutions show that $L \sim c \tau$ for $\tau \gg \tau_{D}$ for all (finite) $\lambda$. The asymptotic rate can be determined by applying this form in Eqn. (7): In this way, the asymptotic rate is approximated as (for $\phi_{f}=1, S_{f \infty}=0$ ),

$$
c=\left(1+\lambda \gamma(n) \sqrt{\tau_{D}}\right)^{-1}, \quad \tau \gg \tau_{D} .
$$

This value is in reasonably good agreement with the numerical results. Hence this asymptotic limit determines the penetration

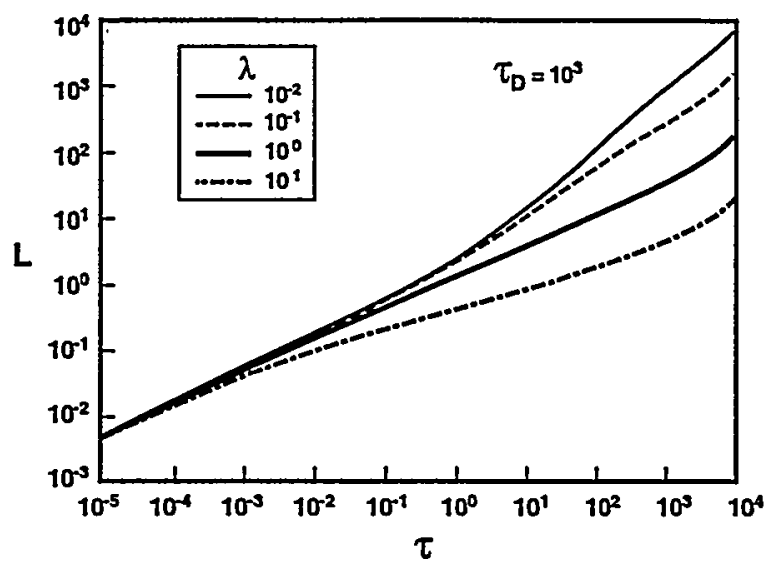

Figure 4. Slug penetration history for finite fracture spacing, $\tau_{D}=10^{3}$, and $n=6$.

distance and rate with good accuracy for $\tau>\tau_{D}$. Using (24) above, the second term on the RHS of Eqn. (25) is determined to be given by the matrix to fracture capacitance ratio,

$$
\frac{C_{m}}{C_{f}}=\lambda \gamma(n) \sqrt{\tau_{D}}=\frac{2 D \phi_{m}\left(S_{w}-S_{m \infty}\right)}{w} .
$$

Hence, the rate is

$$
c=\frac{C_{f}}{C_{f}+C_{m}}
$$

that is, the ratio of available pore volume in the fracture to that in the bulk system. Usually, the aperture is small compared to fracture spacings, thus $c-C_{f} / C_{m}$ because, unless the matrix is very nearly saturated or the matrix porosity is very small (true in some rock systems), $C_{m}$ » $C_{f}$. In the long-time limit, this capacitance ratio determines the rate of advance of the moisture slug in the fracture compared to the saturation rate of the adjoining.intact material.

The cumulative infiltration into the fracture inlet is plotted on Fig. 5 for $\tau_{D}=10^{3}$. These solutions were obtained from a formula similar to Eqn. (20), but taking account of the absorption formula for finite fracture spacing. Note that because the absorption flux at any elevation $z$ decreases at least exponentially fast for elapsed times greater than $\tau_{D}$, the matrix will be saturated over some distance below the fracture inlet. The instantaneous elevation where the matrix is saturated is given approximately by the distance the front travels in time $\tau_{D}, L-c\left(\tau-\tau_{D}\right)$, where $c$ was given in Eqn. (25).

An asymptotic expression, valid in the long-time limit $\tau \gg \tau_{D}$, for the cumulative flux can be determined by using the asymptotic penetration rate for this limit in the flux expression to obtain 


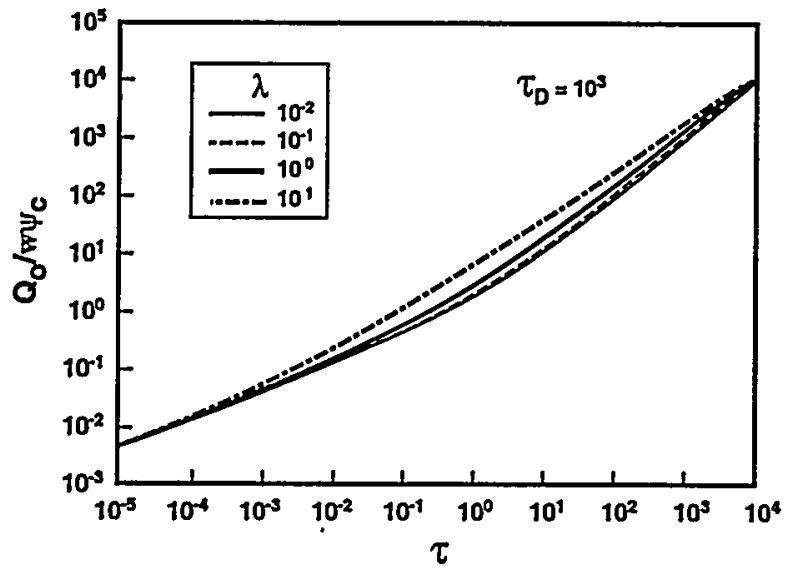

Figure 5. Cumulative absorption of water by the fracture for finite fracture spacing, $\tau_{D}=10^{3}$, and $n=6$.

$$
\frac{Q_{0}}{w \Psi_{c}}=\left(1+2 \frac{C_{m} / C_{f}}{2}\right) c \tau=\tau, \quad \tau \gg \tau_{D}
$$

where the expression for $c$ given in Eqn. (25) is used. The first term in parenthesis on the RHS is the dimensionless imbibed volume residing in the fracture and the second term is the amount residing in the matrix, taking account of material on either side of the fracture. This asymptotic form is identical to the loss-free limit given earlier, and is in good agreement with the numerical values.

\section{CONCLUDING REMARKS}

The parameter $\lambda$ can be viewed as a dimensionless absorption flux parameter. Large values indicate the absorptive flux from fracture to matrix is significant compared to the flux sustainable along the fracture, while small values indicate the opposite. Of significance is that $\lambda$ depends on the square root of the ratio of matrix to fracture diffusivities. Hence, doubling $\lambda$ requires a four-fold relative increase in the matrix diffusivity. To reiterate, in the long-time limit, $\lambda$ determines whether the imbibed moisture resides in the form of long fingers $(\lambda \ll 1)$, or whether it resides in a more-or-less uniform distribution depending mostly on the distance from the wetted surface $(\lambda \gg 1)$. In the long-time limit, the amount imbibed at the surface is independent of $\lambda$, and depends only on the product of fracture transmissivity and time.

Since values of $\lambda$ can be computed a priori for the materials under consideration, these values are useful to determine which geologic units are likely to see significant moisture penetration during an infiltration event. Materials with large values of $\lambda$ will tend to absorb the fracture moisture significantly before allowing extensive transport along fracture pathways. On the other hand, those materials with $\lambda \approx \lambda *$ or smaller will allow significant penetration of fracture moisture, if $\tau \gg \lambda^{-2}$, where $\tau$ indicates the timescale for the infiltration event. Note however that the cumulative amount of moisture imbibed by this system is proportional to $K_{f} w t$ per unit length of fracture, and hence, the cumulative global uptake is the product of $K_{f} t$ and the exposed fracture area on the wetted surface. Since $K_{f} \sim w^{2}$, the cumulative amount is

$$
\chi \frac{A_{\rho} \rho g w^{2} t}{12 \mu}
$$

per unit wetted surface area, where $A_{f}$ is the area ratio taken up by the fractures. The geometric factor $\chi=1$ for finite fracture spacing, and $\chi=\pi / 2$ for semi-infinite spacings, as defined earlier. Thus, while the penetration distance may be extensive for $\lambda \ll 1$, the net amount of moisture transported is only proportional to the fracture area ratio. Nevertheless, the extensive motion of water via fractures can be of significance in problems of contaminant transport, even though the volume of water involved may be small. Martinez (1988) gives a table of values of $\lambda$ for various tuff units believed at that time to be somewhat representative of geologic units at Yucca Mountain, Nevada. Generally, the table shows small values of $\lambda$ for welded tuff units and large values for non-welded units. For a given unit, $\lambda$.decreases with increasing initial moisture content in the matrix, and with increasing fracture aperture. In fact, from the foregoing one can see that $\lambda-w^{-5 / 2}$.

\section{ACKNOWLEDGMENTS}

This work was performed at Sandia National Laboratories. Sandia is a multiprogram laboratory operated by Sandia Corporation, a Lockheed Martin Company, for the United States Department of Energy under Contract DE-ACO4-94AL85000.

\section{APPENDIX}

\section{Numerical Treatment of the Penetration Equation}

The penetration equation (7) is a nonlinear integrodifferential equation with a weakly singular kernel. The singularity is treated by use of interpolants for $s(L)$ in the region near the singularity.

The first step is to discretize the penetration distance and time. We use the notations $L_{i}=L\left(\tau_{i}\right)$ and subdivide the time axis according to $h_{i}=\tau_{i+1}-\tau_{i}$, for $i=1, \ldots, N-1$, where $N-1$ is the total number of time increments computed to the current time $\tau=\tau_{N}$. The task is to compute $L\left(\tau_{N+1}\right)$.

Away from the singularity, we use a trapezoid rule to approximate the integral over the first $N-K$ intervals. Over the 
last $K$ intervals, the integrand involves the so-called Abel kernel (Baker 1977),

$$
I_{l}(\tau-s)=\int_{L_{l}}^{L_{t+1}} \frac{L d L}{\sqrt{\tau-s(L)}}
$$

which is not singular over this interval, but can be badly behaved for $\tau-s\left(L_{t+1}\right) \ll 1$. If we approximate

$$
s(L)=\tau_{l+1} \frac{L-L_{i}}{L_{l+1}-L_{i}}+\tau_{i} \frac{L_{i+1}-L}{L_{i+1}-L_{i}}, \quad L_{i} \leq L \leq L_{i+1},
$$

then the integral is given by

$$
\begin{gathered}
I_{i}(\tau-s)=2\left(L_{i+1}-L_{i}\right)\left(\frac{L_{i+1}}{h_{i}}\left(\Delta \tau_{0}^{1 / 2}-\Delta \tau_{1}^{1 / 2}\right)\right. \\
\left.-\left(\frac{L_{i+1}-L}{3 h_{i}^{2}}\right)\left(2 h_{i}^{3 / 2}-\left(2 \Delta \tau_{1}-h_{i}\right) \Delta \tau_{0}^{1 / 2}\right)\right)
\end{gathered}
$$

where we have used the notation $\Delta \tau_{0}=\tau-\tau_{i}$, and

$\Delta \tau_{1}=\tau-\tau_{i+1}$. The equation is equally valid for $L_{i+1}=L$ and $\tau_{i+1}=\tau$, i.e., for the last interval. In this case,

$$
l_{N}=\frac{2\left(L-L_{N}\right)}{3 \sqrt{h_{N}}}\left(2 L+L_{N}\right), \quad L_{N} \leq L \leq L_{N+1}
$$

Use of these formulae for the integral near the singularity and application of a trapezoid rule over the rest of the region, results in the nonlinear ODE for $L(\tau), \tau_{N} \leq \tau \leq \tau_{N+1}$,

$$
\begin{aligned}
\frac{d L}{d \tau}=1+ & \frac{1}{L}-\frac{\lambda^{N-K}}{L} \sum_{i=1}^{N} l_{i}^{(T)}(\tau-s)-\frac{\lambda}{L} \sum_{i=N-K}^{N-1} I_{i}(\tau-s) \\
& -\frac{2 \lambda}{3 L} \frac{\left(L-L_{N}\right)}{\sqrt{h_{N}}}\left(2 L+L_{N}\right),
\end{aligned}
$$

where $I_{i}^{(T)}$ denotes the trapezoid rule. A start-up algorithm is required which also uses the interpolant formulae. This ODE is integrated forward in time from $\tau=\tau_{N}$ to $\tau=\tau_{N+1}$ using the ODE routines available in the SLATEC mathematics library available on the World-Wide-Web from the Netlib repository (www.netlib.org). We find the Runge-Kutta routine, DERK, works well for $\lambda<1$, and the backward difference routine, DEBDF, works well otherwise. The variable-step trapezoid rule integrals are computed numerically using the routine AVINT in the SLATEC library. This routine is also used to compute the cumulative influx once the penetration has been determined.

\section{REFERENCES}

Abramowitz, M., and I. A. Stegun, Handbook of Mathematical
Functions, Dover Publications, Inc., New York, 1972.

Baker, C. T. H., The Numerical Treatment of Integral Equations, Clarendon Press, Oxford, 1977.

Beven, K., and P., Germann, Macropores and water flow in soils, Water Resources Research, 18, 1311-1325, 1982.

Brooks, R. H., and Corey, A. T., Properties of porous materials affecting fluid flow, ASCE Journal of Irrigation and Drainage Division, 92, 61-88, 1966.

Carslaw, H. S., and J. C. Jaeger, Conduction of Heat in Solids, 2nd. Ed., Clarendon Press, Oxford, 1959.

Gardner, W. R., Some steady-state solutions of the unsaturated moisture flow equation with application to evaporation from a water table, Soil Science, 85 228-232, 1958.

Martinez, M. J., 1988, Capillary-Driven Flow in a Fracture Located in a Porous Medium, Sandia National Laboratories Technical Report, SAND84-1697, Albuquerque, NM.

Nitao, J. J., and Buscheck, T. A., Infiltration of a liquid front in an unsaturated fracture porous medium, Water Resources Research, 27, 2099-2112, 1991.

Philip, J. R., The theory of absorption in aggregated media, Australian Journal of Soil Research, 6 1-19, 1968.

Philip, J. R.,Theory of infiltration, Adv. Hydrosci., 5, 215-296, 1969.

Stakgold, I., Green's functions and boundary value problems, John Wiley \& Sons, New York, 1979.

van Genuchten, M. Th., A closed-form equation for predicting the hydraulic conductivity of unsaturated soils, Soil Science Society of America Journal, 44, 892-898, 1980. 